\title{
SEEstem
}

\section{Wearable navigation device for people with visual impairments}

\author{
Paula Iunes Salles Esteves ${ }^{1}$, Andressa Carmo Pena Martinez ${ }^{2}$, \\ Lucas Francisco da Matta Vegi ${ }^{3}$, Igor Rodrigues Cardoso ${ }^{4}$, \\ Mauro Nacif Rocha ${ }^{5}$, Ricardo dos Santos Ferreira ${ }^{6}$, \\ Denise Mônaco dos Santos ${ }^{7}$ \\ 1,2,3,4,5,6,7 Universidade Federal de Viçosa \\ 1,2,3,4 \{paulaaiunes|andressamartinez|lucasvegi|igor.rodriguescardoso\}@gmail. \\ com $^{5,6,7}\{$ mnacif|ricardo|denise.monaco\}@ufv.br
}

Visually impaired people represent a large amount of the Brazilian population. However, although a wide range of existing legislation ensures accessibility, most of the Brazilian public spaces are inadequate to accommodate disabled citizens. In this context, this paper presents a digital device, which combines the smartphone technologies with Arduino microcontrollers, for orientation and obstacle detection. We tested the minimum viable product and the first vest prototype through a user-centered usability test, which combines HCI assessments to other techniques, such as semi-structured interviews. As known, these wearable devices and mobile applications are in the center of the Internet of Things discussion. This study is expected to be an alternative for the urban mobility of visually impaired people, allowing them to have a more active and independent behavior in public spaces.

Keywords: Assistive wearable devices, Visually impaired people, Accessibility, Human-computer interaction, Collaborative design.

\section{INTRODUCTION}

According to the Brazilian Institute of Geography and Statistics (IBGE, 2011), almost 35 million people declared a type of visual deficiency in Brazil. Visual impairment reduces the ability to identify certain environmental attributes, such as colors, sizes, distances, shapes, and positions, which affect their mobility and interaction in space. To overcome the vision loss, the visually impaired person uses the remainder of the sensory system, to obtain the information for orien- tation and mobility (Dischingher, 2006).

It is clear that the visually impaired represent a large amount of the Brazilian population, which should have the same access and opportunities as other citizens. However, although a wide range of existing legislation ensures accessibility, most of the Brazilian public spaces are inadequate to accommodate disabled citizens. In the country, $42.6 \%$ of the municipalities do not present any accessibility items for people with disabilities (IBGE, 2011). Thus, the 
absence of resources establishes physical and informational barriers, which culminates in their seclusion at home, preventing them from navigating in public spaces.

In this context, this work aims to bring alternatives to fill the lack of adequate infrastructure of the Brazilian public spaces, related to the visually impaired accessibility. The emergence of new digital technologies has brought several possibilities in Architecture and Urbanism, not only for the technological innovation itself, but for making changes in its uses, modes of production, design and manufacture. It is understood that digital technologies are able to reconfigure the navigation in urban spaces, through smaller (miniaturization) and low-cost mobile devices. The development led to the integration of technologies into everyday life, making them more ubiquitous. Therefore, by admitting the hybrid character of spaces, this paper aims to present a digital device development which combines the mobile application with Arduino microcontrollers and sensors, in order to add new layers of information and improve the accessibility of urban spaces for visually impaired pedestrians.

According to Velásquez (2010), there is a variety of Electronic Mobility Aids available to assist visually impaired people, besides traditional resources, such as a cane and a guide dog. Most of the systems are mobile or wearable devices, which allow the user to manipulate the interface and perform another activity while moving through public spaces. These resources can combine several types of inputs and outputs, which provide information about the outdoor environment and warn the user about obstacles.

However, this variety of mobility aids for the visually impaired people does not correspond to the relatively low users' adherence rate. Some researchers believe that the costs, weight, ergonomic problems, and the absence of several functions in the same device are the possible reasons for this lack of acceptance by visually impaired people. Even smartphones have built-in accessibility features and integration with the Google APIs, some of these tech- nologies still have some limitations, such as the inaccuracy of the sensors and a lack of versatility related to the number of sensors and their positions. In this sense, this work incorporates additional resources to mobile devices, through Arduino type microcontrollers, which aims to combine the smartphones technologies for orientation and obstacle detection.

\section{METHODS}

The design of the device involves several challenges, from technological requirements to physical, cognitive and sensory demands related to visually impaired people navigation in urban space. Due to these peculiarities, the interface is a result of a teamwork in a collaborative design process that joined together researchers from the Architecture and Urbanism Department and the Computer Science Department at the Federal University of Viçosa, in Brazil. This multidisciplinary group involves professors and researchers from Nó.Lab (Digital Modeling Lab, related to the Architecture and Urbanism Department) and researchers from LABD2M (Mobile Devices Development Laboratory, based at the Computer Science Department).

The architecture researchers were responsible for the first survey and database collection of tangible user interfaces, the wearable device design and development as well as the definition and application of a user-centered usability test. The computer scientist researchers were responsible for the application development and the Arduino microcontroller integration. However, the group discussed together the schedule, the main activities, and articulated the actions for improving the results in each stage. This multidisciplinary work allows the comprehension and integration of several areas of knowledge, making possible the development of more effective and pleasant interactive systems. The Figure 1 illustrates the workflow activities and teamwork. 
Figure 1

The workflow activities and teamwork

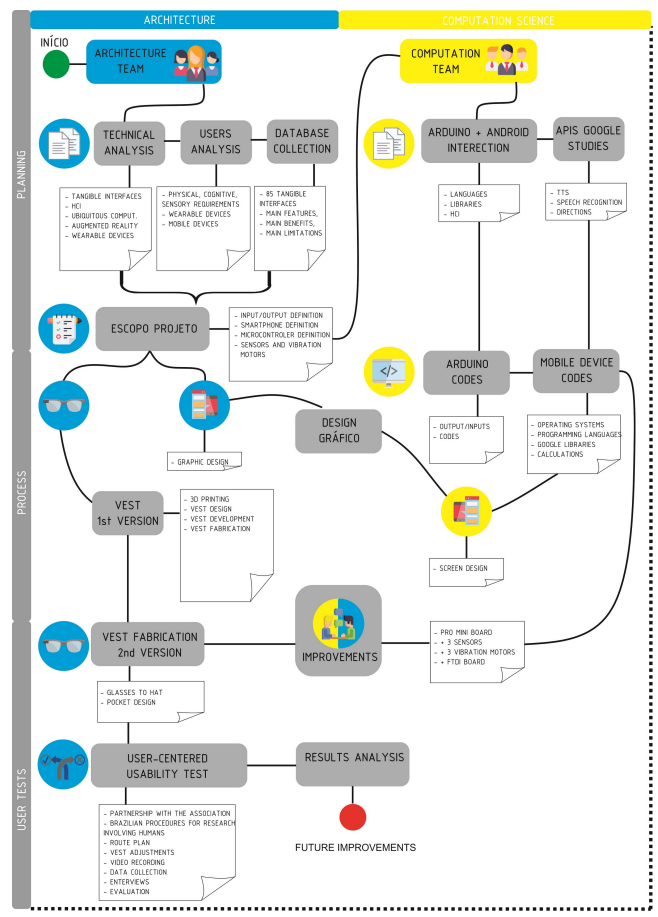

The research is based in five stages:

(A) A survey and database collection of 85 tangible user interfaces related to visually impaired people to analyze and understand their main features, functions, benefits and limitations concerned with user's interaction.

(B) Main Hardware and software components: The device was natively developed for both platforms (Android and iOS operating systems), which corresponded to $99.7 \%$ of the global mobile market in 2016 (GARTNER, 2016). For Android, the application was developed in the integrated development environment (IDE) Android SDK and Android Studio, based on Java and Kotlin programming languages, and the visual interface in the XML language. The iOS operating system uses the Swift language, the visual interface in the XIB language and it was developed in the Xcode integrated development environ- ment (IDE). The minimum mobile requirements for Android operating system is 4.4 KitKat version and 32 $\mathrm{Gb}$ of storage, while the iOS operating system needs a 9 version and $32 \mathrm{~Gb}$ of storage. Additionally, the mobile device also has internet access with the data packet, GPS, microphone, and it can be connected to a headset.

The minimum viable product (MVP) input consists of speech recognition, using Speech Recognizer, in which the user requests a navigation route. The request is transformed into a json format file, converted to a synthesized speech output, developed from the Text to Speech (tts), both libraries maintained by Google. It also needs the Google APIs, such as Google Maps, Google Directions, Google Locations, and Google Places, for receiving navigation information in outdoor public spaces. All of them are the implemented solutions which allow the users to arrive at their destination, informing them when they are within a reasonable distance, the direction (left / right) and angulation of $45^{\circ}, 90^{\circ}$, or $180^{\circ}$, from a "critical point".

(C) Obstacle detection and Arduino microcontroller integration: This stage includes the Arduino microcontroller for obstacle detection through ultrasonic sensors (HC-SR04) and a programming language derived from $\mathrm{C} / \mathrm{C}++$. The ultrasonic sensor transmits the position and the obstacle distance, which activates the vibration motors (1027). The Arduino board is the PRO MINI model (ATMega328), a compact format $(33 \times 18 \times 3 \mathrm{~mm}$ and $3 \mathrm{~g})$ and low-cost board, with $32 \mathrm{~KB}$ memory. The microcontroller has a crystal oscillator of $16 \mathrm{MHz}$, USB connection, reset button, 14 pins of digital input / output, 8 pins of analog inputs (for sensors with variable values), and 6 pins with PWM outputs (for sensors connection). The board can be powered by the USB connection or by an external power supply through the Jack connector. We defined the use of 7 ultrasonic sensors (HCSR04), which detect objects between $2 \mathrm{~cm}$ and 4 meters, with a $3 \mathrm{~mm}$ precision, and 7 vibration motors (1027) for tactile feedback (Figure 2). In addition, we used the FTDI FT232RL card, which allows the con- 
nection of TTL devices to USB.

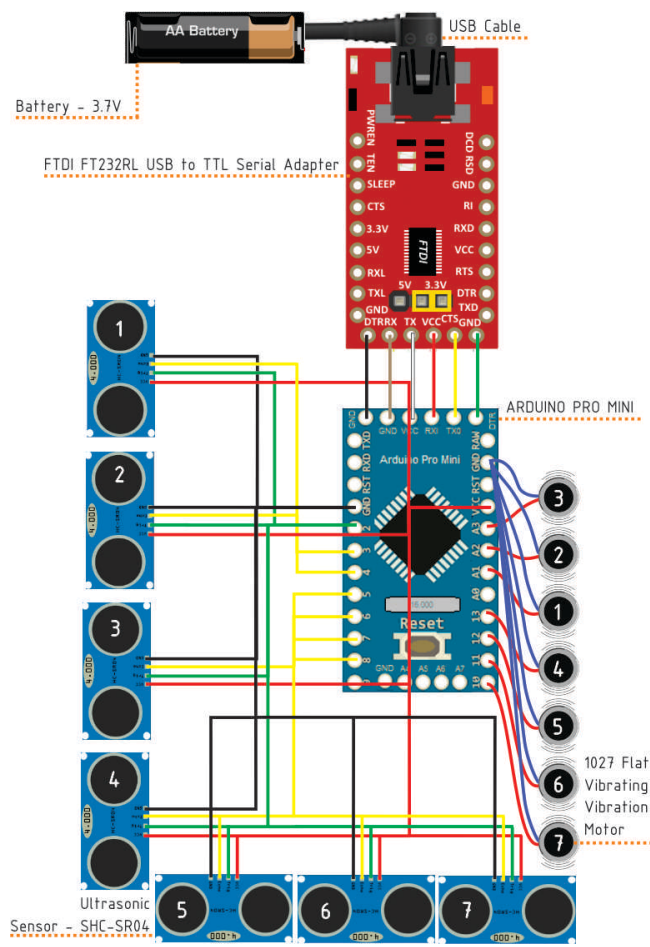

(D) Wearable device development: The vest and the glasses integrate Arduino sensors to assist in obstacle avoidance. There are one Arduino board, five ultrasonic sensors, five vibration motors, connectors, cables, and a power source. The visually impaired person will hardly abandon the cane since it complements the digital device. Therefore, below the waist, the body will be "protected" by the use of the cane, thus the sensors are concentrated in the waist, abdomen and head region.

The vest has four front pockets: the front pocket has an ultrasonic sensor hidden by the cloth. The pockets are attached to the vest by removable Velcro strips, so it is possible to wash the cloth without damaging the components. On the head, the users wear glasses with the ultrasonic sensor and a headset with wireless microphone, joined through 3D printing components, in thermoplastic material. These placements were strategic as they allowed stability for obstacle detection, since there is a natural tendency to keep our head aligned. A 3D printing frame attached to the glasses allow the ultrasonic sensor positions.

For the smartphone location, the researchers proposed an arm phone holder, similar to a sports armband case, in order to keep the user's hands-free for handling the cane. The movements of the arms do not disturb the device operation and its position does not interfere with the sensors' magnetic field. The data transfer between the Arduino microcontroller and the smartphone occurs via USB cable, which can also be used for power charging.

The vest design consists in two stages: the first one in raw cotton fabric, replaced by a second design in gabardine fabric, as well Velcro zipper. The researchers also used some basic sewing tools (sewing machine, thread, needle, etc.).

(E) The user-centered usability test: the tests combined Human-Computer Interaction ( $\mathrm{HCl}$ ) methods for the design and evaluation of user interfaces (UIs), with semi-structured interviews, in a partnership with the Association for the Blind and Visually Impaired people in Juiz de Fora, a Brazilian city. The interface evaluation involved students who take a class for improving the mobility and guidance through digital technologies, at the same partner institution. The researchers planned a walking route nearby the partner institution in Juiz de Fora (on Avenida dos Andradas), observed them and recorded their actions through video recording. The research follows the Brazilian standards and procedures for research involving human subjects and has the ethics committee certificate of approval.

\section{RESULTS \\ The minimum viable product (MVP)}

This paper shows the minimum viable product (MVP), a product with the basic and essential functionalities, in order to validate its usability and provide feedback
Figure 2

The Arduino board, the ultrasonic sensors and vibration motors. 
Figure 3

The vest design (first proposal) for future improvements. As the first steps, the researchers created a wire frame tool, which outlines the main structure of the information flows and visual screens in the mobile application. The interface has three main modules: (1) Information module: describes the direction and angulation that the user should follow; (2) Location module: the user's current position in space and (3) Obstacles Module: distance alert and position of obstacles. The application input consists of speech recognition, using Speech Recognizer, in which the user requests a navigation route.

The Obstacle detection and Arduino microcontroller integration consists of three ultrasonic sensors and three vibration motors. Four pins connect the sensors to the Arduino board: the Vcc, Trigger, Echo and Gnd. The VCC and GND pins are responsible to power the sensor, connecting to the Arduino VCC and GND pins, respectively. The TRIGGER and ECHO pins trigger the ultrasonic signal, and measure the time it returns to the sensor, connecting to the digital pins as input $d$ (pins 3 - 9) and output (pin 2), respectively (Figure 2). After being connected to the board, the sensor starts the scanning by calculating the distance between the object and the user, provided by the ultrasonic library, which reads, transmits data from the sensor and assigns the variables cmMsec and inMsec values of distances.

The formula " $D=P N A * 340 / 2$ " calculates the distance, where " $\mathrm{D}$ " is the distance; "PNA" the pulse at high level; "340 m / s" the ideal speed of sound; and it is divided by 2 , which is the time the sound waves take to go back to the sensor. If the program detects an object at a risky distance to the users, the digital outputs activates the vibration motor, alerting them to the obstacle.

For the wearing device, according to Seymor (2008), wearable computing should prevail for its functionality, as much for its comfort and aesthetics. To this end, the visually impaired person should dress the vest in a versatile way, placing the technology to different locations in the body for detecting the obstacles. Additionally, as the person with visual impairment will hardly abandon the cane, the device will complement its use with sensors in the waist, abdomen and head region (Figure 3 ). The pockets located on the vest have two different parts: The front one, where the ultrasonic sensors are located, partially hidden, and the back of the pocket, where there is a vibration engine in order to be in more contact with the skin, so the user can feel the vibration warnings. The pockets are attached to the dressing by a removable Velcro system, so it is possible to wash the cloth without damaging the components. Additionally, as the vest should not cause discomfort or limit their daily actions (sit, run, walk, among others), there is a front zipper, which allows the user to open or close the vest. The smartphone is held through a similar sports armband case, which keeps the user's hands-free for handling the cane.

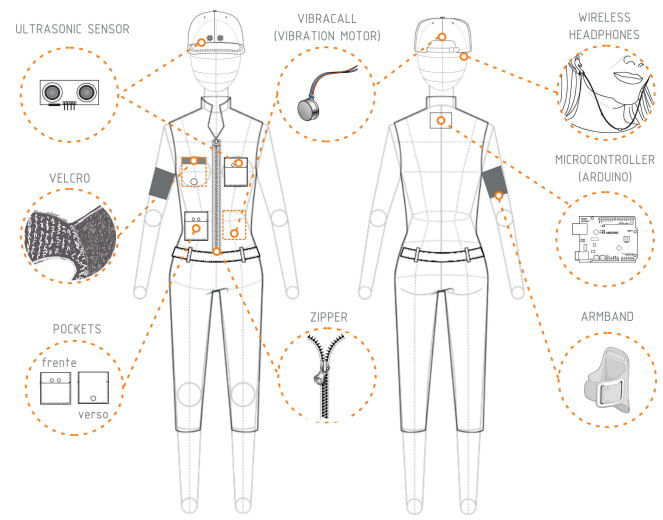

The vest's prototype manufacturing faced some challenges. At the beginning, the wearing device consisted of a vest and a pair of glasses, but the researchers replaced the last one by a hat in order to hide the wires (the electrical circuits, Figure 4). In addition, the more sensors and motors, the more wires are required to connect them in order to activate their settings, which also compromises the aesthetics, since the final volume of wires was higher than initially planned (Figure 5). 


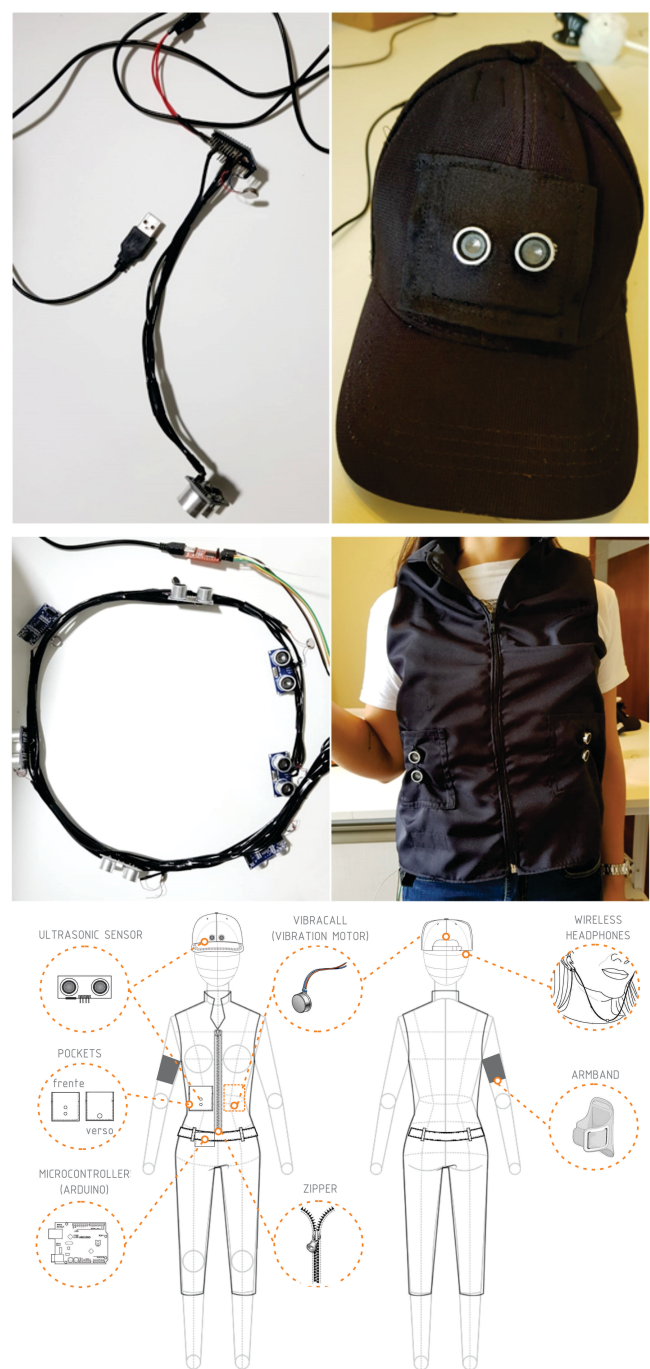

After some tests, the researchers decided to use just a pair of sensors and motors (Figure 6), only in two pockets, due to their position and proximity. The sensor readings in the abdomen are the same of others located in the waist, in such a way that one of them is dispensable. Moreover, some tests showed that more sensors and motors would read the user's own body as a barrier (for example, the arms movement), causing an obstacle avoidance false reading. As a result, we also adjusted the Velcro positions.

The pocket design also changed. Initially the Velcro was intended to connect the pocket with the vest and be removable. However, due to the final Arduino kit volume (board, sensors and jumpers), it would be more feasible to place all the sensors inside the vest. This solution prevents the volume from not compromising the aesthetics and also not bringing discomfort for some users. In order to facilitate washing the cloth, the liner does not have seam in its lower bar, allowing detaching it from the vest. We also observed the fragility and the need to reinforce the vibration motors welding, as there were some rupture during their installation in the vest. However, such issues could be easily repaired, and they do not compromise the cloth manufacture.

\section{The user-centered usability test}

As the device integrates mobile and wearable technologies, as well as tactile and auditory feedbacks, which makes the interaction much more complex, the researchers combined $\mathrm{HCl}$ assessments to other techniques to evaluate the wearable, some of them from Ashbrook et.al (2009). For this evaluation, we performed a user-centered usability test with the students from the Association for the Blind and Visually impaired in Juiz de Fora, Brazil. We observed them and recorded their activities through video recording, along a 400-meter itinerary, previously defined in accordance with the staff of the same institution.

The field test happened on Avenida dos Andradas, in Juiz de Fora - MG, which is the starting point at the "Association of Blind People of Juiz de Fora", and Nossa Senhora da Glória Church as a destination, on the same avenue. This is also a usual route for its students, which could promote greater safety for them while testing the device, as well as the possibility of checking the counterpoints without major challenges.

The institution chose three volunteers to partici-
Figure 4

The pair of glasses replaced by a hat, in order to hide the wires.

Figure 5

The vest and the final volume of wires, higher than initially planned

Figure 6

The vest design after some tests (second proposal) 
Figure 7

User 1 map - first route (red means failures and yellow obstacle detection)

Figure 8

User 1 map - second route (red means failures and yellow obstacle detection)

Figure 9

User 2 map - first route (red means failures and yellow obstacle detection) pate in this experiment: two blind men (a 34-year-old and a 68-year-old men) and one low vision woman (51 years old). Following the Research Ethics Committee regulations, we replaced their names by a sequence from 1 to 3 to respect the anonymity of the users throughout the entire evaluation process. The test began at 2:00 pm and surveyors completed the 400 meters in an average of 6 minutes. For a better representation of the information, we generated user-centered maps, which show the identified and unidentified obstacles, complemented by pictures. In the maps, it is possible to observe the activity of the device in the three users.

We reported different outcomes, according to the user. The first user, for example, (Figure 07 and 08) wears vest sizes bigger than the others, and the cloth adjustment in place did not provide the comfort, nor the maximum potential of the vest, since one of the sensors ended up hidden. In addition, this user walked faster than the others, and most of the time the obstacle warning occurred when the user was already very close to the obstacle.

The user number 2, (Figure 09 and 10), detected less obstacle than the others, probably as she is not completely blind (low vision), she can divert from some obstacles before she even approaches them. User number 3 map (Figure 11 and 12) is the one who presents the highest frequency of detection, such as walls and bigger obstacles. At the end, the device detected $59 \%$ obstacles, failing $41 \%$ of the time. We considered detection failure when the device did not trigger the warning in time to avoid it. Sometimes the device could not detect tiny and thin objects, such as the poles near the destination, as well as some objects below the user's waist (dumpsites), since there were no sensors in that region.
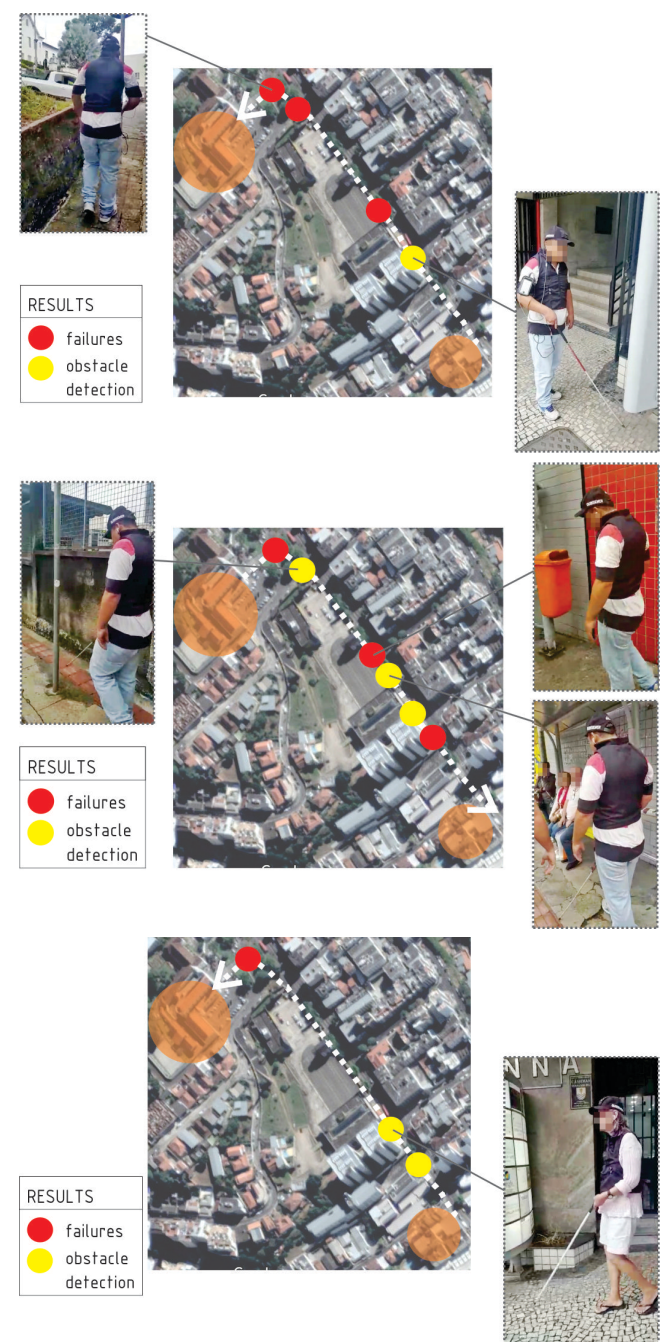


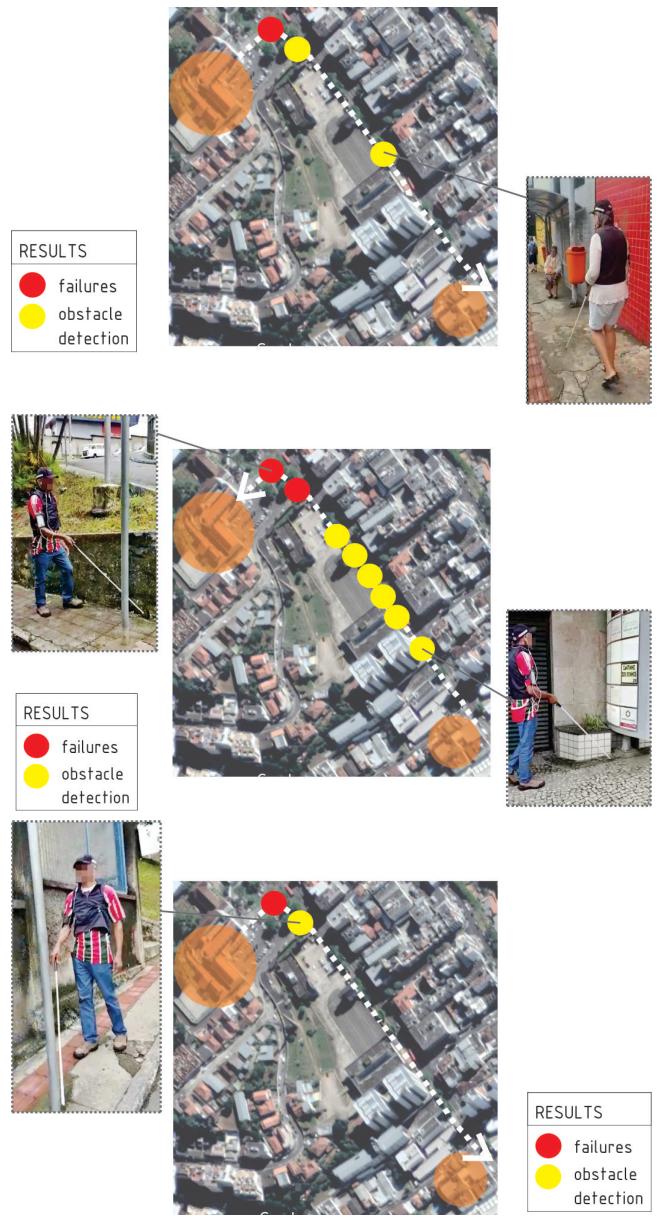

Although the smartphone application functioned properly, it also did not show the expected result. The straight-line test detected a lack of voice instructions when there was no changing in the route, in such a way that some users considered it to be an application bug. The application should send more positioning information, in shorter periods of time, to ensure they were on the right path.

After the tests, we conducted semi-structured interviews to understand each users' experience. The user number 1 praised the initiative and felt no difficulty in the device usability. However, he pointed out some flaws, as described above, and stated the device should be improved so that he could re-use it with confidence. He stated that adding more sensors would bring discomfort to the user and suggested improving only the existing system without adding any extra item to the wearable. The second interviewee liked the device and the sensations it provides. During the conversation, she showed a superior degree of satisfaction compared to the previous interviewee and did not present many complaints about the device. However, she stated some discomfort with digital devices, and she would need to get familiar with the technology to use it again with confidence. In addition, in contrast to the first interviewee, she believes it would be interesting to add more sensors.

Finally, the third interviewee also praised the initiative and the device, even if it needs more improvements. During the interview, the user stated that he walked along the street more carefully than normal since he had never used the device before. However, despite reporting some shortcomings, the interviewee believes he would use the device again. As a final suggestion, he states that the number of sensors is ideal, and their increase could bring some degree of discomfort.

All the volunteers and staff from the Association of Blind People were concerned about the final cost of the device, emphasizing the need for an affordable value for its commercialization. They also requested for traffic light detection as a possible improvement.

\section{DISCUSSIONS AND IMPROVEMENTS}

The results show that more than two sensors compromises the aesthetics of the cloth, due to the final volume of wires, as well as increases the complexity to use the device. The design improvement and cloth remodeling advance towards the miniaturization, similar to the head module (Figure 13). The dressing will consist of an adjustable pouch/collar, where the sensors and vibration motors would be
Figure 10

User 2 map - second route (red means failures and yellow obstacle detection)

Figure 11

User 3 map - first route (red means failures and yellow obstacle detection)

Figure 12

User 3 map - second route (red means failures and yellow obstacle detection) 
Figure 13

The pocket (left) and head modules (right), after miniaturization

Figure 14

The design improvement and cloth remodeling: the adjustable pouch

Figure 15

The design improvement and cloth remodeling: the adjustable collar arranged in their extension, and the microcontroller and its battery would fit inside the existing pocket (Figure 14 and Figure 15). Such changes would improve the vest flexibility of use, which allows changing the positioning according to the user's needs, also solving the size adjustment problems related to body types. In addition, we will replace the ultrasonic sensor to a different type, such as a laser, to reduce the margin of error and achieve the expected accuracy.
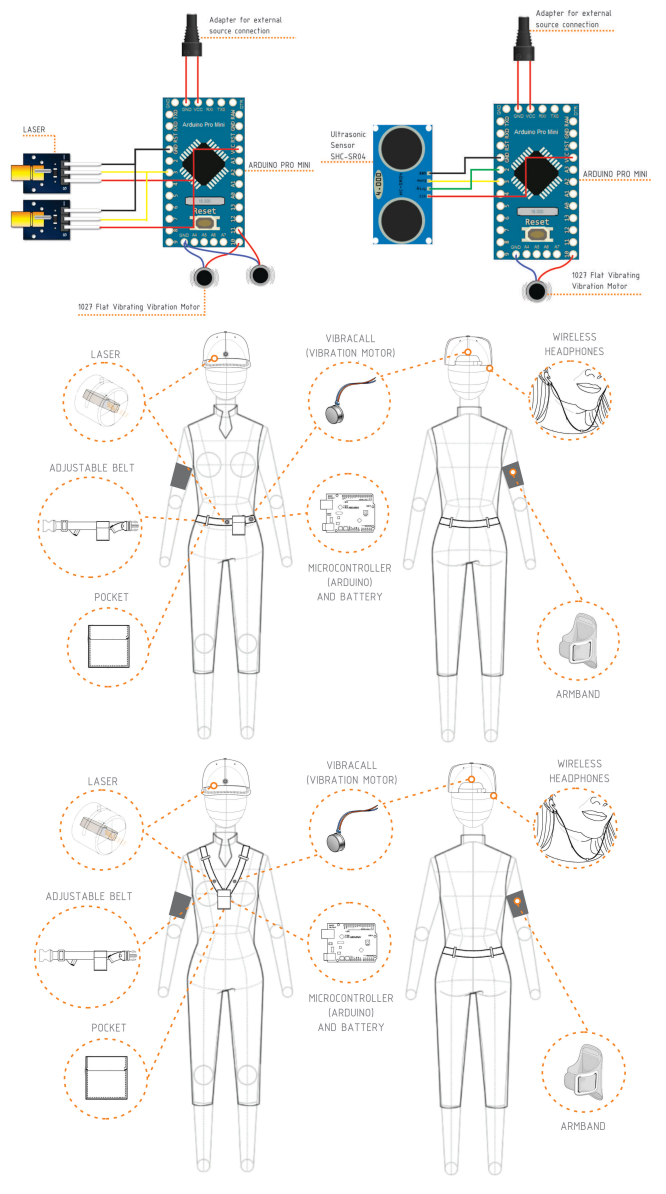

After the user-centered tests, the researchers had already made some changes to the mobile application. They migrated the Google's APIS use to Open Street Maps (OSM), which is completely free and offers some other advantages, such as pedestrian routes, and the inclusion of voluntary geographic information (VGI). Thus, the OSM enables the user to draw sidewalks and even pedestrian tracks, as well as to instruct them, even when there are no direction changes, just confirming their path from time to time, adding directions such as "go ahead", "missing" or "about $15 \mathrm{~m}$ to the destination". We believe it will solve the problems detected during the usercentered usability test, which will improve the user's confidence on the application and allow a more reliable and safer route.

We also added a tool that allows the device to detect the traffic signals through the camera, which recognize two images: one in which the green color is evidenced, and another one in which the red color is in evidence. This generates a Gaussian or Laplacian pyramid, which only highlights what is already in evidence, obfuscating the rest. The map will emit which color has the highest amount in the camera capture, and if the result indicates the red color, the application concludes the traffic light is closed; if indicates the green color, then the semaphore is open.

As known, these wearable devices and mobile applications are in the center of the Internet of Things discussion. This study is expected to be an alternative for the urban mobility of visually impaired people, allowing them to have a more active and independent behavior in public spaces.

\section{ACKNOWLEDGE}

We thank the Cnpq (Conselho Nacional de Desenvolvimento Científico e Tecnológico) for the undergraduate scientific initiation grant and the Graduate Program in Architecture and Urbanism at UFV (Federal University of Viçosa) for supporting this work. We extend the appreciation to the students at the Association of Blind People of Juiz de Fora, for accepting to participate and collaborate for the improvements in this work. 


\section{REFERENCES}

Bakker, S, Hausen, D, SELKER, T, Van Der Hoven, E, Butz, A and Eggen, B 2014 'Peripheral Interaction: Shaping the Research and Design Space', Proceedings of the CHI 2014 workshop

Daniel, A, Kent, L, James, C and Thad, S 2009, '9 Methods of Evaluation for Wearable Computing', in Cho, Gilsoo (eds) 2009, Smart clothing - technology and applications, CRC Press, Boca Raton, pp. 229-257

Dischinger, M 2006, 'The Non-Careful Sight', in Devlieger, P, Renders, F, Froyen, $\mathrm{H}$ and Wildiers, K (eds) 2006, Blindness and the Multi-sensorial City, Garant Uitgevers N V, Garant, Antwerpen, pp. 143-176

Instituto Brasileiro de Geografia e Estatística, IBGE (eds) 2011 a, Censo Demográfico 2010, Ministério do Planejamento, Orçamento e Gestão, Rio de Janeiro

Instituto Brasileiro de Geografia e Estatística, IBGE (eds) 2011 b, Perfil dos Municípios Brasileiros, Ministério do Planejamento, Orçamento e Gestão, Rio de Janeiro

Kassim, A, Yasuno, T, Suzuki, H, Shahrieel, M, Izzuan, H, Jafar, F and Subramonian, S 2016, 'Conceptual design and Implementation of Electronic Spectacle Based Obstacle Detection for Visually Impaired Persons', Journal of Advanced Mechanical Design, Systems, and Manufacturing, 10, pp. 1-12

Monteiro, A, Silva, E and Lopes, I 2014, 'Localização para Deficientes Visuais Utilizando um Gadget para Orientação', Revista Innover, 1, pp. 93-105

Panëels, SA, Varenne, D, Blum, JR and Cooperstock JR 2013 'The Walking Straight Mobile Application: Helping the Visually Impaired Avoid Veering', Proceedings of International Conference on Auditory Display, ICAD, pp. 25-32

Ramiro, V 2010, 'Wearable Assistive Devices for the Blind' in Lay-Ekuakille, A and Mukhopadhyay, SC (eds) 2010, Wearable and Autonomous Biomedical Devices and Systems for Smart Environment: Issues and Characterization, Springer, Berlin, pp. 331-349

Sabine, S (eds) 2008, Fashionable Technology: the intersection of design, fashion, science and technology Springer Publishing Company, Vienna

[1] https://www.gartner.com/newsroom/id/3415117 